\title{
Whole genome sequencing-based detection of antimicrobial resistance and virulence in non-typhoidal Salmonella enterica isolated from wildlife
}

\author{
Milton Thomas ${ }^{1,2}$, Gavin John Fenske ${ }^{1,2}$, Linto Antony ${ }^{1,2}$, Sudeep Ghimire ${ }^{1,2}$, Ronald Welsh³, \\ Akhilesh Ramachandran ${ }^{3^{*}}$ and Joy Scaria ${ }^{1,2^{*}}$ (B)
}

\begin{abstract}
The aim of this study was to generate a reference set of Salmonella enterica genomes isolated from wildlife from the United States and to determine the antimicrobial resistance and virulence gene profile of the isolates from the genome sequence data. We sequenced the whole genomes of 103 Salmonella isolates sampled between 1988 and 2003 from wildlife and exotic pet cases that were submitted to the Oklahoma Animal Disease Diagnostic Laboratory, Stillwater, Oklahoma. Among 103 isolates, 50.48\% were from wild birds, 0.9\% was from fish, 24.27\% each were from reptiles and mammals. 50.48\% isolates showed resistance to at least one antibiotic. Resistance against the aminoglycoside streptomycin was most common while 9 isolates were found to be multi-drug resistant having resistance against more than three antibiotics. Determination of virulence gene profile revealed that the genes belonging to csg operons, the fim genes that encode for type 1 fimbriae and the genes belonging to type III secretion system were predominant among the isolates. The universal presence of fimbrial genes and the genes encoded by pathogenicity islands 1-2 among the isolates we report here indicates that these isolates could potentially cause disease in humans. Therefore, the genomes we report here could be a valuable reference point for future traceback investigations when wild life is considered to be the potential source of human Salmonellosis.
\end{abstract}

Keywords: Wildlife, Salmonellosis, Whole genome sequencing, Antimicrobial resistance, Salmonella virulence, Foodborne pathogen

\section{Background}

Salmonella enterica is the leading cause of foodborne illness in the United States accounting for approximately 1.2 million infections, 23,000 hospitalizations and 450 deaths annually. Over the past few decades, Salmonella has acquired new virulence determinants that influence host-tropism which helps these organisms to adapt to a wide range of hosts [1]. Multiple serovars of S. enterica

\footnotetext{
*Correspondence: rakhile@okstate.edu; joy.scaria@sdstate.edu ${ }^{1}$ Department of Veterinary and Biomedical Sciences, South Dakota State University, Brookings, SD 57007, USA

${ }^{3}$ Oklahoma Animal Disease Diagnostic Laboratory, Oklahoma State University, Stillwater, OK 74078, USA

Full list of author information is available at the end of the article
}

originating from mammalian, reptilian and avian hosts have been reported to cause infections in humans [1]. Wildlife and exotic pets harboring Salmonella are potential sources for human infections [1]. Transmission of Salmonella from wildlife and exotic animals to humans occurs through multiple pathways. Increasing evidence suggests that there could be a bidirectional transmission of Salmonella between domesticated and wild animals. Farm animals acquiring Salmonella from wildlife could increase the risk of human infection. Salmonella infections in humans have also been reported through direct contact with exotic pets and wildlife, especially those in captivity. Consumption of contaminated game bird meat is also a potential source for foodborne salmonellosis. 
Furthermore, wildlife such as rodents and birds, harboring in the proximity of food production units can act as carriers and contaminate food products leading to indirect infections.

The threat posed by salmonellosis is further compounded by the presence of resistance genes that confer resistance to multiple antimicrobial drugs. According to the National Antimicrobial Resistance Monitoring System (NARMS) integrated report, 20\% of human Salmonella isolates exhibit antimicrobial resistance (AMR). Antimicrobial-resistant Salmonella infections result in increased disease severity and longer hospitalizations in addition to economic losses [2]. Research indicates that Salmonella isolates from various wildlife species also possess AMR determinants and the prevalence rate of AMR genes in these isolates could be as high as $100 \%$ [3, 4]. Thus, Salmonella in wildlife poses a significant risk to human health underlining the need for an integrative 'One Health' approach for the surveillance of pathogens among humans, domestic animals, and wildlife population.

Whole genome sequencing (WGS) of foodborne pathogens could be adopted as an effective and rapid surveillance tool. Compared to conventional antimicrobial tests, WGS offers a more comprehensive information on the genotypic characteristics of pathogens including identification of AMR and virulence determinants, and serotypes. Recent studies have utilized WGS to reliably predict the antimicrobial characteristics in various pathogens including Salmonella [5-8]. In this study, WGS was utilized to predict AMR and virulence determinants in Salmonella isolated from exotic pets and wildlife.

\section{Methods}

\section{Quality assurance}

All strains were identified as Salmonella enterica following the American Association of Veterinary Laboratory Diagnosticians certified laboratory. For genome sequencing, each isolate was streaked on Salmonella selective medium and a single colony was picked and used for further steps as outlined below.

\section{Salmonella bacterial isolates}

A total of 103 Salmonella isolates were revived from archival cultures obtained from exotic pet or wildlife clinical specimens submitted to the Oklahoma Animal Disease Diagnostic Laboratory, Stillwater, Oklahoma during 1988-2003. The metadata for the samples used in this study is provided in Table 1 and the details of genome sequencing and assembly parameters are given in Additional file 1: Table S1. Isolates were streaked on Luria-Bertani agar slants and were transported to the Animal Disease Research and Diagnostic Laboratory,
South Dakota State University, Brookings, South Dakota for WGS. Samples were streaked on Luria-Bertani agar plates upon arrival to the laboratory. A single bacterial colony from the agar plate was then inoculated to LuriaBertani broth and cultured at $37^{\circ} \mathrm{C}$.

\section{Genomic DNA isolation and WGS}

Genomic DNA was isolated from $1.0 \mathrm{~mL}$ overnight cultures using the Qiagen DNeasy kits (Qiagen, Valencia, CA, USA) according to manufacturer's protocol. The quality of isolated DNA was analyzed using NanoDrop ${ }^{\mathrm{TM}}$ One (Thermo Scientific ${ }^{\mathrm{TM}}, \mathrm{DE}$ ) and was quantified using Qubit $^{\circledR} 3.0$ (Thermo Fisher Scientific Inc., MA) fluorometer and stored at $-20{ }^{\circ} \mathrm{C}$ until use. Whole-genome sequencing was performed on Illumina Miseq platform using V2 chemistry with $2 \times 250$ paired-end chemistry Briefly, the concentrations of genomic DNA samples were adjusted to $0.3 \mathrm{ng} / \mu \mathrm{L}$ concentration and were processed using Nextera XT DNA Sample Prep Kit (Illumina Inc. San Diego, CA). The libraries were normalized using bead-based procedure and pooled together at equal volume. The pooled library was denatured and sequenced using Miseq reagent version 2 (Illumina, Inc., CA).

\section{Genome assembly and identification of resistance and virulence genes}

The raw data files were de-multiplexed and converted to FASTQ files using Casava v.1.8.2. (Illumina, Inc, San Diego, CA). The FASTQ files were trimmed and assembled de novo using CLC Genomics workbench 9.4 (Qiagen Bioinformatics, CA). The antibiotic resistance genes in the assembled Salmonella genomes were identified by BLAST search against a local copy of the antibiotic resistance gene sequence data from ResFinder [9] and CARD [10]. The parameters used for BLAST search were $\geq 95 \%$ gene identity and $50 \%$ sequence length of the resistance gene. The virulence genes in the genomes were predicted using a similar approach. Salmonella virulence gene sequences were extracted from Virulence Factor Database [11] and Salmonella genome assemblies were searched against these sequences using BLAST with $\geq 90 \%$ gene identity and $50 \%$ sequence length cut off.

\section{Serotyping and antimicrobial susceptibility test}

Serotypes of the strains were determined at the National Veterinary Service Laboratory, Ames, IA. Antimicrobial susceptibility of all Salmonella isolates was determined using the Sensititre NARMS Gram Negative Plate (CMV3AGNF, Thermofisher). The antibiotics used were gentamicin, streptomycin, amoxicillin-clavulanic acid, ampicillin, cefoxitin, ceftiofur, ceftriaxone, azithromycin, chloramphenicol, nalidixic acid, ciprofloxacin, 
Table 1 List of Salmonella enterica strains isolated and sequenced from wild life and the corresponding metadata

\begin{tabular}{|c|c|c|c|c|c|}
\hline Strain ID & Serovar & Year & Animal & NCBI SRA BioSample ID & NCBI SRA ID \\
\hline ADRDL-001 & Poona & 1993 & Alligator omentum & SAMN06330630 & SRR5278825 \\
\hline ADRDL-002 & Typhimurium & 1993 & Auodad feces & SAMN06330629 & SRR5278822 \\
\hline ADRDL-003 & Gaminara & 1994 & Ratite intestine & SAMN06330628 & SRR5278823 \\
\hline ADRDL-004 & Lille & 1993 & Gamebird embryo & SAMN06330627 & SRR5278827 \\
\hline ADRDL-005 & Typhimurium & 1993 & Ratite feces & SAMN06333495 & SRR5278819 \\
\hline ADRDL-006 & Typhimurium & 1993 & Ratite feces & SAMN06333494 & SRR5278824 \\
\hline ADRDL-007 & Thompson & 1993 & Ratite cecum & SAMN06333493 & SRR5278802 \\
\hline ADRDL-008 & Livington & 1993 & Ratite cecum & SAMN06333492 & SRR5278806 \\
\hline ADRDL-009 & Typhimurium & 1993 & Ratite feces & SAMN06333491 & SRR5278801 \\
\hline ADRDL-010 & Montevideo & 1993 & Ratite feces & SAMN06333489 & SRR5278805 \\
\hline ADRDL-011 & 6,7-nonmotile & 1993 & Ratite intestine & SAMN06333488 & SRR5278804 \\
\hline ADRDL-012 & Arechavaleta & 1994 & Ratite intestine & SAMN06333486 & SRR5278803 \\
\hline ADRDL-013 & 4,5,12:i-monophasic & 1994 & Ratite liver & SAMN06333485 & SRR5380966 \\
\hline ADRDL-014 & Berta & 1994 & Ratite intestine & SAMN06333484 & SRR5278808 \\
\hline ADRDL-015 & Ituri & 1994 & Ratite cecum & SAMN06333483 & SRR5278773 \\
\hline ADRDL-016 & Ituri & 1994 & Ratite intestine & SAMN06333482 & SRR5278772 \\
\hline ADRDL-017 & Heidelberg & 1993 & Wild turkey liver & SAMN06333481 & SRR5278779 \\
\hline ADRDL-018 & Heidelberg & 1993 & Wild turkey liver & SAMN06333480 & SRR5278777 \\
\hline ADRDL-019 & Godesberg & 1993 & Wild turkey cecum & SAMN06333479 & SRR5278778 \\
\hline ADRDL-020 & 4,5,12:i-monophasic & 1993 & Eclectus colon & SAMN06333477 & SRR5278771 \\
\hline ADRDL-021 & Anatum & 1993 & Giraffe feces & SAMN06333476 & SRR5278774 \\
\hline ADRDL-022 & Anatum & 1993 & Giraffe feces & SAMN06333475 & SRR5278780 \\
\hline ADRDL-023 & Pomona & 1993 & Python abdominal swab & SAMN06333473 & SRR5278767 \\
\hline ADRDL-024 & Muenchen & 1993 & Ratite intestine & SAMN06333472 & SRR5278776 \\
\hline ADRDL-025 & Typhimurium & 1994 & Rodent intestine & SAMN06333471 & SRR5278770 \\
\hline ADRDL-026 & Hadar & 1995 & Wild chicken intestine & SAMN06333470 & SRR5278768 \\
\hline ADRDL-027 & Hadar & 1994 & Ratite intestine & SAMN06333469 & SRR5278769 \\
\hline ADRDL-028 & Typhimurium & 1988 & Primate intestine & SAMN06333465 & SRR5278873 \\
\hline ADRDL-029 & Albany & 1988 & Saiga intestine & SAMN06333464 & SRR5278882 \\
\hline ADRDL-030 & Arizona & 1988 & Snake & SAMN06333462 & SRR5330438 \\
\hline ADRDL-031 & Arizona & 1989 & Boa intestinal swab & SAMN06333460 & SRR5330446 \\
\hline ADRDL-032 & 16:z10-e,n,xz15 & 1989 & Cervine feces & SAMN06333459 & SRR5330441 \\
\hline ADRDL-033 & Enteritidis & 1989 & Hedgehog spleen & SAMN06333458 & SRR5330440 \\
\hline ADRDL-034 & Typhimurium $(\mathrm{O} 5-)^{*}$ & 1992 & Pigeon airsac swab & SAMN06333457 & SRR5330448 \\
\hline ADRDL-035 & Typhimurium & 1989 & Screech owl liver & SAMN06333455 & SRR5330445 \\
\hline ADRDL-036 & Braenderup & 1989 & Snow leopard intestine & SAMN06333454 & SRR5330444 \\
\hline ADRDL-037 & Saintpaul & 1989 & Snow leopard lung & SAMN06333453 & SRR5330406 \\
\hline ADRDL-038 & Montevideo & 1992 & Cervid intestine & SAMN06333451 & SRR5329403 \\
\hline ADRDL-039 & Enteriditis & 1993 & Emu feces & SAMN06333450 & SRR5329404 \\
\hline ADRDL-040 & Enteriditis & 1993 & Emu feces & SAMN06333449 & SRR5380965 \\
\hline ADRDL-041 & Worthington & 1992 & Quail intestine & SAMN06333448 & SRR5380958 \\
\hline ADRDL-042 & $\begin{array}{l}\text { II 43:z4,z23:- or IIla 43:z4,z23:- or } \\
\text { Farmingdale or IV 43:z4,z23:-* }\end{array}$ & 1992 & Reptile eggsac & SAMN06333447 & SRR5329405 \\
\hline ADRDL-043 & Panama & 1992 & Rhea intestine & SAMN06333694 & SRR5409894 \\
\hline ADRDL-044 & Ituri & 1994 & Ratite cecum & SAMN06333692 & SRR5409893 \\
\hline ADRDL-045 & Newport & 1995 & Ratite feces & SAMN06333691 & SRR5409493 \\
\hline ADRDL-046 & Newport & 1995 & Dolphin lung & SAMN06333689 & SRR5409890 \\
\hline ADRDL-047 & Typhimurium & 1997 & Psittacine lung & SAMN06333684 & SRR5409485 \\
\hline ADRDL-048 & Typhimurium & 1997 & Psittacine intestine & SAMN06333683 & SRR5409315 \\
\hline ADRDL-049 & Muenchen & 1996 & Ratite intestine & SAMN06333682 & SRR5409313 \\
\hline
\end{tabular}


Table 1 continued

\begin{tabular}{|c|c|c|c|c|c|}
\hline Strain ID & Serovar & Year & Animal & NCBI SRA BioSample ID & NCBI SRA ID \\
\hline ADRDL-050 & Schwazengrund & 1997 & Ratite intestine & SAMN06333681 & SRR5409312 \\
\hline ADRDL-051 & Archavaleta & 1997 & Antelope intestine & SAMN06333692 & SRR5409893 \\
\hline ADRDL-052 & Infantis & 1997 & Fish water & SAMN06645614 & SRR5398012 \\
\hline ADRDL-053 & Bredeney & 1998 & Llama intestine & SAMN06333861 & SRR5409360 \\
\hline ADRDL-054 & Plymouth & 1997 & Reptile liver & SAMN06330627 & SRR5278827 \\
\hline ADRDL-055 & Montevideo & 1997 & Reptile intestine & SAMN06645663 & SRR5398013 \\
\hline ADRDL-056 & Branderup & 1995 & Wild chicken intestine & SAMN06645590 & SRR5387496 \\
\hline ADRDL-057 & Enteriditis & 1996 & Wild chicken intestine & SAMN06645569 & SRR5387492 \\
\hline ADRDL-058 & Typhimurium & 1996 & Wild chicken feces & SAMN06645567 & SRR5387491 \\
\hline ADRDL-059 & Bredeney & 1995 & Gamebird intestine & SAMN06645592 & SRR5387497 \\
\hline ADRDL-060 & Livingston & 1996 & Gamebird intestine & SAMN06645590 & SRR5387496 \\
\hline ADRDL-061 & Enteriditis & 1995 & Psittacine intestine & SAMN06645588 & SRR5387490 \\
\hline ADRDL-062 & Montevideo & 1996 & Psittacine liver & SAMN06645587 & SRR5387493 \\
\hline ADRDL-063 & 7,14:K-monophasic & 1995 & Ratite intestine & SAMN06645585 & SRR5387523 \\
\hline ADRDL-064 & Anatum & 1995 & Ratite feces & SAMN06645654 & SRR5387521 \\
\hline ADRDL-065 & Enteriditis & 1995 & Ratite & SAMN06645582 & SRR5387527 \\
\hline ADRDL-066 & Thompson & 1995 & Ratite cloacal swab & SAMN06645594 & SRR5387519 \\
\hline ADRDL-067 & Thompson & 1995 & Ratite cloacal swab & SAMN06645593 & SRR5387517 \\
\hline ADRDL-068 & $4,5,12: \mathrm{i}$ & 1995 & Ratite pericardial fluid & SAMN06645652 & SRR5387518 \\
\hline ADRDL-069 & Livingston & 1996 & Llama intestine & SAMN06645650 & SRR5387514 \\
\hline ADRDL-070 & Uganda & 1999 & Cervine intestine & SAMN06645664 & SRR5398014 \\
\hline ADRDL-071 & Lille & 2000 & Cervine intestine & SAMN06645663 & SRR5398013 \\
\hline ADRDL-072 & Parera & 1998 & Iguana cloacal swab & SAMN06645662 & SRR5398016 \\
\hline ADRDL-073 & Anatum & 1998 & Ratite feces & SAMN06645661 & SRR5398025 \\
\hline ADRDL-074 & Anatum & 1998 & Ratite feces & SAMN06645615 & SRR5398018 \\
\hline ADRDL-075 & Kiambu & 1998 & Ratite cloacal swab & SAMN06645614 & SRR5398012 \\
\hline ADRDL-076 & Marina & 2000 & Reptile feces & SAMN06645660 & SRR5398017 \\
\hline ADRDL-077 & Bredeney & 2003 & Alpaca liver & SAMN06645613 & SRR5398015 \\
\hline ADRDL-078 & Sandiego & 2003 & Alpaca feces & SAMN06645612 & SRR5398009 \\
\hline ADRDL-079 & Sandiego & 2003 & Alpaca feces & SAMN06645611 & SRR5398010 \\
\hline ADRDL-080 & Bredeney & 2003 & Antelope feces & SAMN06645610 & SRR5398011 \\
\hline ADRDL-081 & Virginia or Muenchen* & 2002 & Ratite & SAMN06645609 & SRR5398008 \\
\hline ADRDL-082 & Newport* & 2002 & Ratite & SAMN06645659 & SRR5398007 \\
\hline ADRDL-083 & Enteritidis* & 2002 & Ratite & SAMN06645658 & SRR5398001 \\
\hline ADRDL-084 & Oranienburg & 2003 & Iguana cloacal swab & SAMN06645657 & SRR5398006 \\
\hline ADRDL-085 & Give & 2003 & Iguana cloacal swab & SAMN06658957 & SRR5409330 \\
\hline ADRDL-086 & Chameleon & 2003 & Iguana cloacal swab & SAMN06333875 & SRR5387539 \\
\hline ADRDL-087 & Typhimurium & 2002 & Llama feces & SAMN06333874 & SRR5387538 \\
\hline ADRDL-088 & Anatum & 2003 & Llama feces & SAMN06333873 & SRR5387533 \\
\hline ADRDL-089 & Typhimurium & 2003 & Llama feces & SAMN06333872 & SRR5387534 \\
\hline ADRDL-090 & Agona & 2003 & Marsupial intestine & SAMN06333871 & SRR5387532 \\
\hline ADRDL-091 & Miami & 2001 & Reptile fecal swab & SAMN06658960 & SRR5409328 \\
\hline ADRDL-092 & Arizona & 2001 & Reptile liver & SAMN06658959 & SRR5409327 \\
\hline ADRDL-093 & & 2001 & Reptile cloacal swab & SAMN06658958 & SRR5409325 \\
\hline ADRDL-094 & Marina & 2002 & Reptile cloacal swab & SAMN06658962 & SRR5409322 \\
\hline ADRDL-095 & Marina & 2002 & Reptile abscess swab & SAMN06658961 & SRR5409324 \\
\hline ADRDL-096 & Arizona & 2002 & Reptile lung & SAMN06333869 & SRR5387526 \\
\hline ADRDL-097 & Parera & 2002 & Reptile cloacal swab & SAMN06333866 & SRR5397979 \\
\hline ADRDL-098 & Chameleon & 2002 & Reptile cloacal swab & SAMN06333865 & SRR5397978 \\
\hline ADRDL-099 & Senftenberg & 2002 & Reptile cloacal swab & SAMN06333864 & SRR5397977 \\
\hline
\end{tabular}


Table 1 continued

\begin{tabular}{|c|c|c|c|c|c|}
\hline Strain ID & Serovar & Year & Animal & NCBI SRA BioSample ID & NCBI SRA ID \\
\hline$\overline{\mathrm{ADRDL}-100}$ & Arizona & 2002 & Reptile cloacal swab & SAMN06333863 & SRR5409363 \\
\hline ADRDL-101 & Arizona & 2002 & Reptile cloacal swab & SAMN06333862 & SRR5409361 \\
\hline ADRDL-102 & Kisarwe & 2003 & Reptile cloacal swab & SAMN06333861 & SRR5409360 \\
\hline ADRDL-103 & Newport & 2003 & Turtle intestine & SAMN06333859 & SRR5409359 \\
\hline
\end{tabular}

* Predicted serovar using Seqsero

sulfisoxazole, trimethoprim-sulfamethoxazole, and tetracycline. The AMR was determined according to Clinical and Laboratory Standards Institute guidelines except for azithromycin and sulfisoxazole where the data obtained was indeterminate and were not included in further analysis.

\section{Results and discussion \\ Distribution of Salmonella isolates among wildlife and exotic pets}

A total of 103 Salmonella isolates sampled between 1988 and 2003 from wildlife and exotic pets were included in the present study for determining the antimicrobial susceptibility using whole genome sequencing. Among 103 isolates, 52 isolates $(50.48 \%)$ were from wild birds, 1 isolate $(0.9 \%)$ was from fish, 25 isolates each $(24.27 \%)$ were from reptiles and mammals (Table 1$)$. The serovars of 96 isolates in this study were determined at the National Veterinary Service Laboratory, Ames, IA, and the remaining 6 serovars were predicted using Seqsero [12]. The serovar of one isolate (ADRDL-093) was not identified under Kauffmann-White classification. A total of 45 serovars were identified among the 103 isolates, of which Typhimurium (12.62\%) was the most frequent serovar. Other serovars that had higher prevalence were Enteritidis (6.8\%), Anatum (5.8\%), Arizona (5.8\%), Bredeney (3.9\%) and Montevideo (3.9\%). The presence of multiple serotypes in wildlife has also been reported from previous epidemiological studies. Nine Salmonella samples isolated from marine mammals and birds in California yielded 7 serovars [4]. Similar to our findings, Salmonella Typhimurium was reportedly the predominant serovar present in wildlife [13-15] in various parts of the world.

\section{Phenotypic resistance to antimicrobials}

Antimicrobial susceptibility test of 103 Salmonella bacterial isolates was performed using Sensititre NARMS gram-negative plate. The results were classified into 3 categories: resistant, intermediate, or susceptible. Fiftytwo out of the 103 isolates (50.48\%) showed resistance to at least one antibiotic (Fig. 1a). Resistance against the aminoglycoside streptomycin was most commonly observed. Forty-eight of the 103 isolates (46.6\%) exhibited this phenotype. However, only three isolates (2.9\%) showed resistance to gentamicin which also belonged to the aminoglycoside class of antibiotics. The isolates with resistance against gentamicin were also resistant to streptomycin. In the beta-lactam group, ampicillin resistance was the most common phenotype and was seen in 11 of the isolates $(10.67 \%)$. Among these 11 isolates, few also shared resistance against other beta-lactams such as amoxicillin-clavulanic acid (4), cefoxitin (3), and ceftiofur (3). All the isolates were susceptible to ceftriaxone except one with intermediate resistance. The isolates that were susceptible to ampicillin were also susceptible to all other beta-lactams. Chloramphenicol resistance was observed for seven isolates (6.7\%), trimethoprim-sulfamethoxazole resistance in $4(3.88 \%)$ and tetracycline resistance in $19(18.44 \%)$ of the isolates. All the isolates were susceptible to ciprofloxacin and all except one isolate was susceptible to nalidixic acid. Nine isolates were found to be multi-drug resistant having resistance against more than three antibiotics.

\section{Genotypic resistance to antimicrobials}

The presence of genes that could contribute to AMR was detected by BLAST searching the assembled Salmonella genomes against a local copy of Resfinder and CARD sequence data (Fig. 1b). Additional details on the query length and percentage of gene identity for the BLAST results are provided in Additional file 2: Table S2. Bacterial isolates showing "intermediate" resistance on antimicrobial susceptibility test was grouped with "susceptible" isolates for the calculation of sensitivity and specificity of AMR genotype. Twenty-two genes that provided resistance to aminoglycosides were detected and the genes were present in 100 isolates. The sensitivity was $100 \%$ and specificity was $5.45 \%$ for resistance against aminoglycosides. The low specificity was probably due to the lack of resistance genes being expressed in vitro. Genes responsible for resistance to beta-lactam antibiotics were detected in 11 isolates which were also resistant by antimicrobial susceptibility test. The plasmid-mediated cephalosporinase gene blaLAT-1, plasmid-borne class $\mathrm{C}$ beta-lactamase gene blaBIL-1, and blaCMY (Class C) genes were found together and were detected in three isolates. Genes belonging to blaTEM (class A) were found in eight isolates. Collectively, there 
a

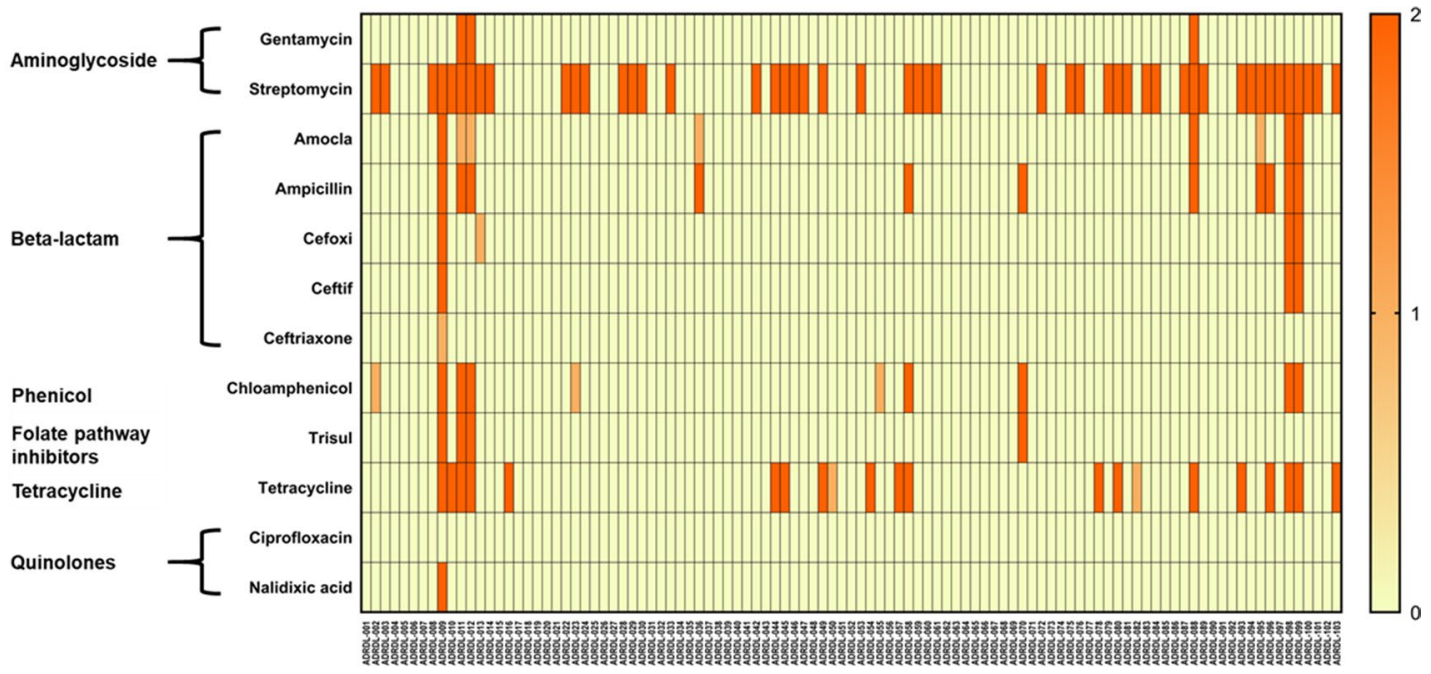

b

Antibiotics class $\left\{\begin{array}{l}\text { A } \\ \text { A }\end{array}\right.$

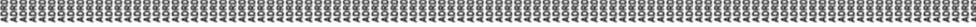

Multi-drug efflux pump promoter

Multi-drug efflux
pump

Multi-drug efflux pump regulator

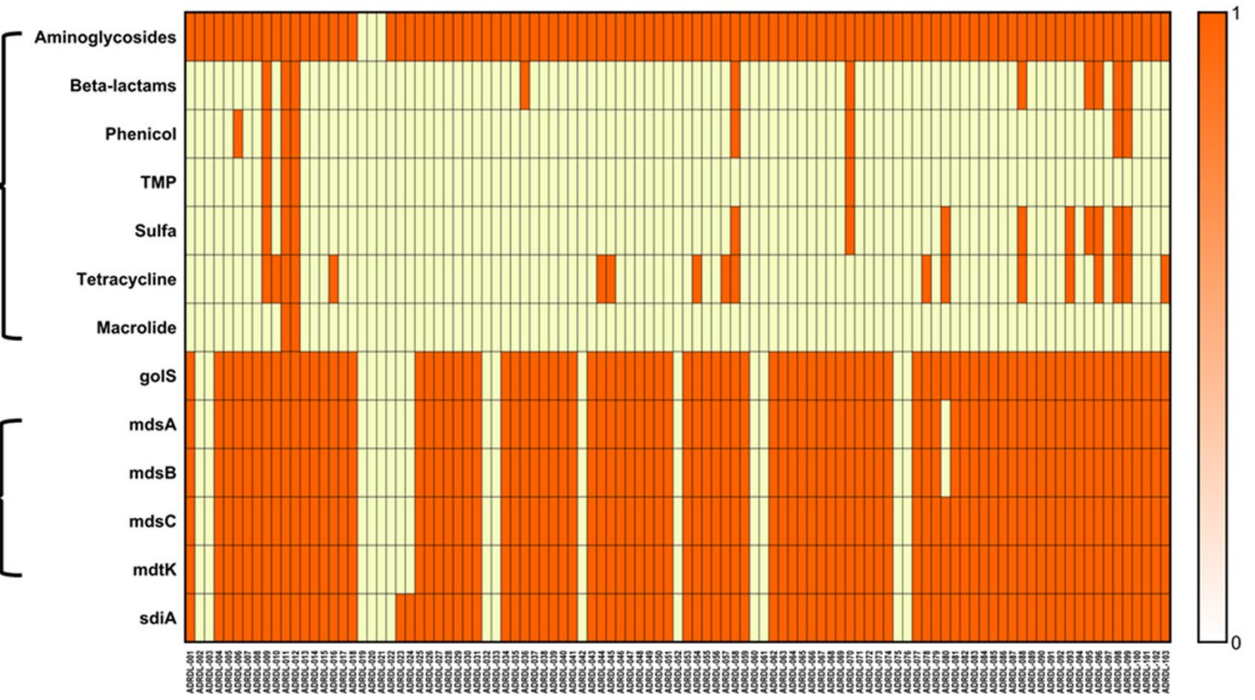

Fig. 1 Phenotypic and genotypic anti-microbial resistance of 103 wildlife salmonella isolates. a Heatmap of phenotypic resistance against 12 antibiotics was measured using Sensititre NARMS gram-negative plate. The CLSI breakoff points for resistance against various antibiotics was used for determining the antimicrobial susceptibility of 103 salmonella strains. Legend description: $0=$ susceptible, $1=$ intermediate, and $2=$ resistant b Heatmap of genotypic resistance against antimicrobials detected using CLC workbench 9.0 by BLAST against ResFinder 2.1 and CARD database. Legend description: $0=$ absent, 1 = present. The golS, mdsABC complex, and mdtK genes associated with multidrug resistance was present in all except 16 isolates. Similarly, multidrug efflux pump regulator gene sdiA was absent in 14 isolates. Complete data used to generate $\mathbf{b}$ is given in Additional file 2: Table S2

were 280 beta-lactamase genes present in those 11 isolates. The sensitivity and specificity was $100 \%$ for betalactams. Phenicol resistance encoded by cat, catA1, and floR genes was present in 8 isolates. The sensitivity was $100 \%$ and specificity was $98.96 \%$ for phenicol resistance. $d f r A 1, d f r A 10, d f r A 12$, sul1, sul2, and sul3 genes conferring resistance to trimethoprim-sulfamethoxazole drugs were present in 12 isolates. The sensitivity was $100 \%$ and specificity was $91.92 \%$ for trimethoprim-sulfamethoxazole. The sul1, sul2, and sul3 genes could also contribute to resistance against sulfisoxazole. However, a definite conclusion of genotype-phenotype correlation is lacking due to the absence of antimicrobial susceptibility test data that matches the CLSI recommended breakpoint for resistance against sulfisoxazole. Tetracycline resistance encoded by tet $(A), \operatorname{tet}(B), \operatorname{tet}(C)$, and $\operatorname{tet}(D)$ genes for tetracycline efflux pumps were detected in 18 samples all of which were also resistant by antimicrobial susceptibility test. The sensitivity was $94.74 \%$ and specificity was $100 \%$ for tetracycline resistance. Two isolates carried the $m p h(A)$ gene which confers resistance to macrolides. However, the only macrolide that was tested 
was azithromycin and the genotype-phenotype relation could not be established due to lack of data from antimicrobial susceptibility test that matches with the breakpoint recommended by CLSI (>32 $\mathrm{mg} / \mathrm{L}$ ).

Overall, the sensitivity for detecting AMR using genotype was $100 \%$ except for tetracycline where 1 isolate was phenotypically resistant even in the absence of the (tet) gene. The specificity for aminoglycosides had the highest degree of incongruence between genotype and phenotype. Fifty-two isolates that were positive for aminoglycoside resistance genes were phenotypically susceptible. Although not to the degree found in this study, a mismatch in phenotype-genotype correlation was also reported previously in E. coli and Salmonella for aminoglycoside resistance, especially for streptomycin [5, 16]. There was $100 \%$ phenotype-genotype correlation for beta-lactam resistance. Phenicols and tetracycline also had $>98 \%$ specificity, while trimethoprim-sulfamethoxazole had lower specificity (91.2\%) because of four isolates that were genotypically resistant but were phenotypically susceptible. These results are also similar to those obtained in previous studies $[5,16]$ where correlation approaching $100 \%$ was obtained for antimicrobials other than aminoglycosides.

In addition to the genes that confer AMR, we also analyzed the genes that could confer multi-drug resistance (Fig. 1b). The golS gene is a promoter for multidrug efflux pump, mdsABC [17] and was detected among 84.46\% ( $\mathrm{n}=87$ ) isolates. Similarly, mdsABC (multidrug transporter of Salmonella) complex which is made up of mdsA, mdsB, and mdsC units, was found in all isolates that had gols gene except one isolate which lacked $m d s B$ and $m d s C$ genes. The mdsABC complex is known to provide resistance against a variety of drugs and toxins and is involved in Salmonella virulence and pathogenicity [17, 18]. The $m d t K$ gene, a multi-efflux pump which could provide resistance against norfloxacin, doxorubicin and acriflavin [18] and $s d i A$, a regulator for multi-drug resistance pump AcraB [19], were present in 84.46 and $86.41 \%$ of the isolates respectively. The presence of these genes could contribute to the virulence and pathogenicity of these Salmonella isolates and also indicates the potential for these isolates to resist various antibiotics and toxins.

\section{Analysis of virulence determinants}

The genes that are associated with virulence among 103 wildlife Salmonella isolates were analyzed (Fig. 2) using CLC workbench 9.4. The parameters used were the minimum identity of $90 \%$ and minimum length of $50 \%$. Additional details on the query length and percentage of gene identity for the BLAST results are given in Additional file 3: Table S3. A total of 197 virulence genes were detected by BLAST search against a local copy of the Virulence Factor Database. The virulence-associated determinants

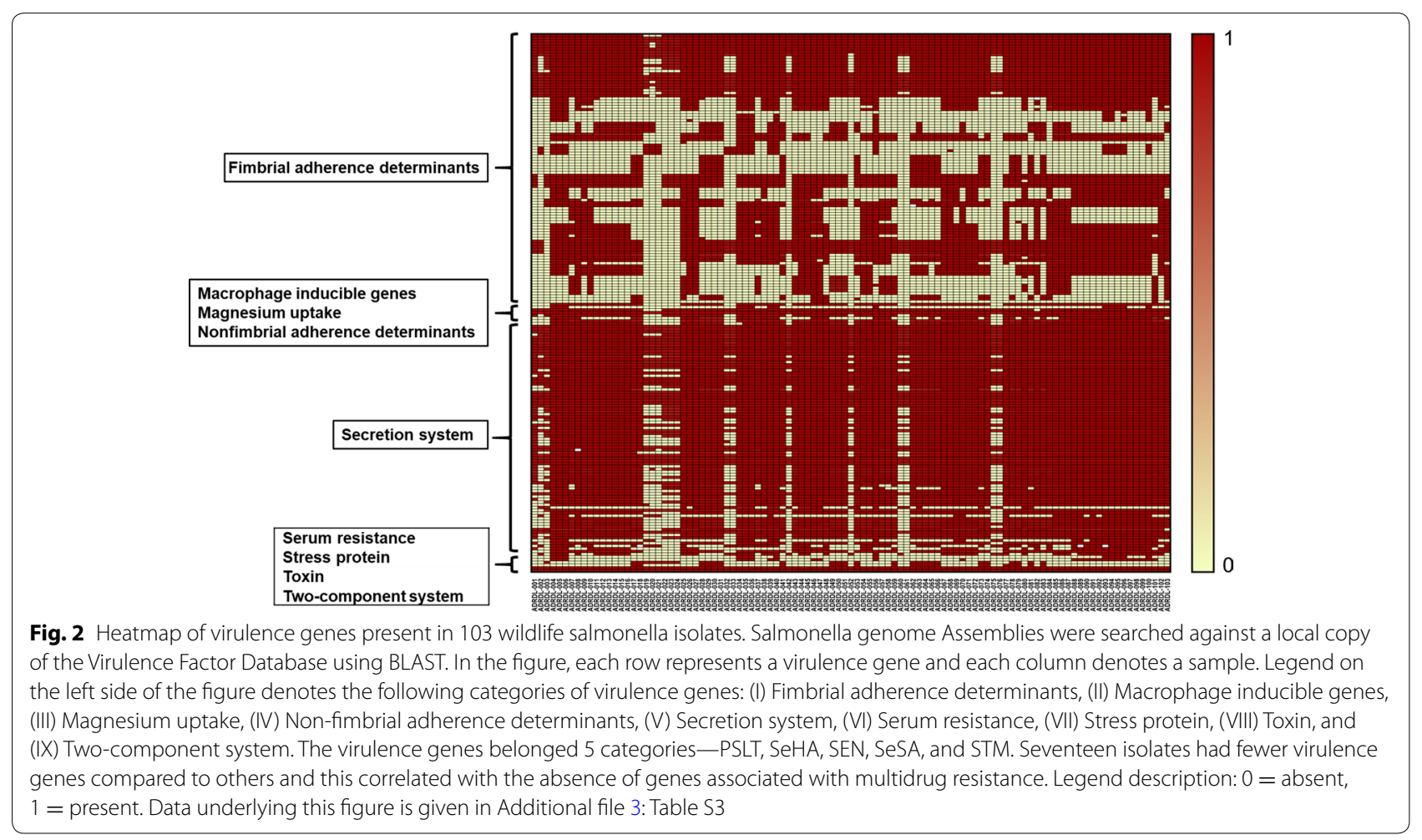


collectively were grouped under 9 categories: fimbrial adherence determinants, macrophage inducible genes, determinants associated with magnesium uptake, nonfimbrial adherence determinants, genes associated with secretion system, serum resistance determinants, stress proteins, toxins, and two-component regulatory systems.

Among fimbrial adherence determinants, the genes belonging to two csg operons $\operatorname{csg} B A C$ and $\operatorname{csg} D E F G$ were present universally in all isolates. These genes encode for curli fimbriae or thin aggregative fimbriae and mediate binding to various serum and tissues matrix proteins [20]. Another gene cluster that was ubiquitously present were the fim genes that encodes for type 1 fimbriae. This cluster is comprised of the fimAICDHF operon and three regulatory genes fim W, fim $Y$, and fim $Z$ and mediates adherence to eukaryotic cells [21]. However, the fim $Y$ gene was not detected in ten isolates at the BLAST search cut-off level we used.

The genes belonging to type III secretion system (TTSS/T3SS) encoded by Salmonella pathogenicity island-1 (SPI-1) and -2 (SPI-2) were also predominantly present among the isolates. This included SPI-1 regulator genes hilACD, and SPI-1 encoded inv/spa, and prg/org operons that were detected in all the isolates. Similarly, SPI-2 regulatory gene $s s r B$, chaperone protein-encoding genes $-s s c A$ and $s s c B$, and $s s a$ genes that encode for T3SS2 apparatus were also present among 103 isolates. However, the sse genes which encode for the effectors were observed only in fewer isolates. Another set of genes that were present in all isolates were the genes that respond to magnesium level in the extracellular environment [22]. This included mgtc, which mediates magnesium uptake and $p h o P-p h o Q$ genes that are regulators of the two-component system.

The least abundant virulence determinants were the $t c f$, $s t a$, and pef fimbrial operons and spv gene cluster. These genes belonging to the fimbrial adherence determinants category were detected in less than $25 \%$ of the isolates. Additionally, $r c k$ gene that provides protection against the complement-mediated immune response of the host was also found in low abundance. There were 16 isolates that possessed fewer than $50 \%$ of the total virulence genes in the database (Fig. 2). These isolates include ADRDL002, -003, -019, -020, -021, -022, -023, -024, -032, -033, $-042,-052,-060,-061,-075$, and -076 . Importantly, these isolates also had a lower abundance of genes that contributed to multi-drug resistance (Fig. 1). However, these isolates come under various serotypes and were isolated from different host species. Therefore, a common factor responsible for the observed low abundance of virulence genes is not evident. The universal presence of fimbrial genes and the genes encoded by pathogenicity islands 1-2 among the isolates we report here indicates that these isolates could potentially cause disease in humans. Therefore, the genomes we report here could be a valuable reference point for future traceback investigations in instances where wildlife may be considered as a potential source of human Salmonellosis.

\section{Additional files}

Additional file 1: Table S1. Details on genome sequencing and assembly parameters. Salmonella genome assemblies were performed using CLC workbench v 9.4. Quality control of the sequencing data and genome assembly metrics (number of contigs, N50 value, and genome coverage) for each genome is listed.

Additional file 2: Table S2. Genotypic Antibiotic resistance gene profile of 103 Salmonella isolates. Salmonella genome assemblies were searched against a local copy of the Resfinder database using BLAST. Cut off parameter for BLAST search was $\geq 95 \%$ gene identity and 50\% sequence length of the resistance gene. Values in the sample columns indicate the BLAST sequence percentage identity cutoff values.

Additional file 3: Table S3. Mapping of virulence genes present in 103 wild life salmonella isolates. Salmonella genome assemblies were searched against a local copy of the Virulence Factor Database using BLAST.

Minimum identity of $90 \%$ and minimum length of $50 \%$ BLAST hits were used as cut off value. Reference column indicates the NCBI gene locus tag of the reference genes used. Values in the sample columns indicate the BLAST sequence percentage identity cutoff values.

\section{Abbreviations}

AMR: antimicrobial resistance; NARMS: The National Antimicrobial Resistance Monitoring System; WGS: whole genome sequencing

\section{Authors' contributions}

JS and AR conceived and designed the study. MT, GJF, LA and SG performed the experiments. RW originally developed the culture archive. MT analyzed the data. MT, JS and AR wrote the manuscript. All authors read and approved the final manuscript.

\section{Author details}

${ }^{1}$ Department of Veterinary and Biomedical Sciences, South Dakota State University, Brookings, SD 57007, USA. ${ }^{2}$ South Dakota Center for Biologics Research and Commercialization, Brookings, SD 57007, USA. ${ }^{3}$ Oklahoma Animal Disease Diagnostic Laboratory, Oklahoma State University, Stillwater, OK 74078, USA.

\section{Acknowledgements}

Authors thank the Section of Bacteriology, Animal Disease Research and Diagnostic Laboratory, South Dakota for helping with the antimicrobial susceptibility testing of the Salmonella isolates. We also thank Scott Talent and Leanne Tillman (Oklahoma Animal Disease Diagnostic Laboratory, Stillwater, OK) for reviving archival cultures necessary for this study.

\section{Competing interests}

The authors declare that they have no competing interests.

\section{Availability of data and materials}

Genome sequence data of 103 Salmonella enterica isolates have been submitted to NCBI Sequence Read Archive (NCBI SRA) for public access. NCBI SRA accession number for 103 isolates described in this manuscript is given in Table 1.

Consent for publication

All authors gave the consent for publication.

Ethics approval and consent to participate Not applicable. 


\section{Funding}

This work was supported in part by the USDA National Institute of Food and Agriculture, Hatch Projects SD00H532-14 and SD00R540-15, and the United States Food and Drug Administration GenomeTrakr project subcontract to awarded JS. The funding agencies had no role in study design, data collection, and interpretation, or the decision to submit the work for publication.

\section{Publisher's Note}

Springer Nature remains neutral with regard to jurisdictional claims in published maps and institutional affiliations.

Received: 27 June 2017 Accepted: 6 November 2017

Published online: 21 November 2017

\section{References}

1. Hoelzer K, Switt Al, Wiedmann M. Animal contact as a source of human non-typhoidal salmonellosis. Vet Res. 2011;42:34.

2. Krueger AL, et al. Clinical outcomes of nalidixic acid, ceftriaxone, and multidrug-resistant nontyphoidal salmonella infections compared with pansusceptible infections in FoodNet sites, 2006-2008. Foodborne Pathog Dis. 2014;11(5):335-41.

3. Botti $V$, et al. Salmonella spp. and antibiotic-resistant strains in wild mammals and birds in north-western Italy from 2002 to 2010. Vet Ital. 2013;49(2):195-202.

4. Smith WA, Mazet JA, Hirsh DC. Salmonella in California wildlife species: prevalence in rehabilitation centers and characterization of isolates. J Zoo Wildl Med. 2002;33(3):228-35

5. McDermott PF, et al. Whole-genome sequencing for detecting antimicrobial resistance in nontyphoidal Salmonella. Antimicrob Agents Chemother. 2016;60(9):5515-20.

6. Bradley $\mathrm{P}$, et al. Rapid antibiotic-resistance predictions from genome sequence data for Staphylococcus aureus and Mycobacterium tuberculosis. Nat Commun. 2015;6:10063.

7. Metcalf BJ, et al. Using whole genome sequencing to identify resistance determinants and predict antimicrobial resistance phenotypes for year 2015 invasive pneumococcal disease isolates recovered in the United States. Clin Microbiol Infect. 2016;22(12):1002e1-8.
8. Metcalf BJ, et al. Short-read whole genome sequencing for determination of antimicrobial resistance mechanisms and capsular serotypes of current invasive Streptococcus agalactiae recovered in the USA. Clin Microbiol Infect. 2017;23(8):574.e7-574.e14

9. Zankari $\mathrm{E}$, et al. Identification of acquired antimicrobial resistance genes. J Antimicrob Chemother. 2012;67(11):2640-4.

10. Jia B, et al. CARD 2017: expansion and model-centric curation of the comprehensive antibiotic resistance database. Nucleic Acids Res. 2017:45(D1):D566-73.

11. Chen L, et al. VFDB 2012 update: toward the genetic diversity and molecular evolution of bacterial virulence factors. Nucleic Acids Res. 2012;40(Database issue):D641-5.

12. Zhang $S$, et al. Salmonella serotype determination utilizing high-throughput genome sequencing data. J Clin Microbiol. 2015;53(5):1685-92.

13. Refsum T, et al. Salmonellae in avian wild life in Norway from 1969 to 2000. Appl Environ Microbiol. 2002;68(11):5595-9.

14. Gopee NV, Adesiyun AA, Caesar K. Retrospective and longitudinal study of salmonellosis in captive wildlife in Trinidad. J Wildl Dis. 2000;36(2):284-93.

15. Chambers DL, Hulse AC. Salmonella serovars in the herpetofauna of Indiana County, Pennsylvania. Appl Environ Microbiol. 2006;72(5):3771-3.

16. Tyson $\mathrm{GH}$, et al. WGS accurately predicts antimicrobial resistance in Escherichia coli. J Antimicrob Chemother. 2015;70(10):2763-9.

17. Pontel LB, et al. GolS controls the response to gold by the hierarchical induction of Salmonella-specific genes that include a CBA efflux-coding operon. Mol Microbiol. 2007;66(3):814-25.

18. Nishino K, Latifi T, Groisman EA. Virulence and drug resistance roles of multidrug efflux systems of Salmonella enterica serovar Typhimurium. Mol Microbiol. 2006;59(1):126-41.

19. Rahmati $S$, et al. Control of the AcrAB multidrug efflux pump by quorumsensing regulator SdiA. Mol Microbiol. 2002;43(3):677-85.

20. Romling U, et al. Curli fibers are highly conserved between Salmonella typhimurium and Escherichia coli with respect to operon structure and regulation. J Bacteriol. 1998;180(3):722-31.

21. Zeiner SA, Dwyer BE, Clegg S. FimA, FimF, and FimH are necessary for assembly of type 1 fimbriae on Salmonella enterica serovar Typhimurium. Infect Immun. 2012;80(9):3289-96.

22. Groisman EA. The pleiotropic two-component regulatory system PhoPPhoQ. J Bacteriol. 2001;183(6):1835-42.

\section{Submit your next manuscript to BioMed Central and we will help you at every step:}

- We accept pre-submission inquiries

- Our selector tool helps you to find the most relevant journal

- We provide round the clock customer support

- Convenient online submission

- Thorough peer review

- Inclusion in PubMed and all major indexing services

- Maximum visibility for your research

Submit your manuscript at www.biomedcentral.com/submit
BioMed Central 